\title{
Two Explicit Characterizations of the General Nonnegative-Definite Covariance Matrix Structure for Equality of BLUEs, WLSEs, and LSEs
}

\author{
Phil D. Young ${ }^{1}$, Joshua D. Patrick ${ }^{2} \&$ Dean M. Young ${ }^{2}$ \\ ${ }^{1}$ Department of Information Systems and Business Analytics, Baylor University, Waco, TX \\ ${ }^{2}$ Department of Statistical Science, Baylor University, Waco, TX \\ Correspondence: Phil D. Young, Department of Information Systems and Business Analytics, Baylor University, Waco, \\ TX 76798, USA. Tel: 1-254-710-7394. E-mail: philip_young @baylor.edu
}

Received: June 30, 2020 Accepted: October 11, 2020 Online Published: October 21, 2020

doi:10.5539/ijsp.v9n6p108 URL: https://doi.org/10.5539/ijsp.v9n6p108

\begin{abstract}
We provide a new, concise derivation of necessary and sufficient conditions for the explicit characterization of the general nonnegative-definite covariance structure $\boldsymbol{V}$ of a general Gauss-Markov model with $E(\boldsymbol{y})$ and $\operatorname{Var}(\boldsymbol{y})$ such that the best linear unbiased estimator, the weighted least squares estimator, and the least squares estimator of $\boldsymbol{X} \boldsymbol{\beta}$ are identical. In addition, we derive a representation of the general nonnegative-definite covariance structure $\boldsymbol{V}$ defined above in terms of its Moore-Penrose pseudo-inverse.
\end{abstract}

Keywords: matrix equations, orthogonal-projection matrices, matrix column space, matrix rank, Moore-Penrose pseudoinverse

\section{Introduction}

We consider the general Gauss-Markov model

$$
\boldsymbol{y}=\boldsymbol{X} \boldsymbol{\beta}+\epsilon,
$$

where $\boldsymbol{y}$ is an $n \times 1$ vector of observations, $\boldsymbol{X}$ is an $n \times p$ known fixed, non-null model (design) matrix such that $\operatorname{rank}(\boldsymbol{X})=p$, $\boldsymbol{\beta}$ is a $p \times 1$ vector of unknown model parameters, and $\boldsymbol{\epsilon}$ is an $n \times 1$ vector of random perturbations such that $E(\boldsymbol{\epsilon})=\mathbf{0}_{n \times 1}$ and $\operatorname{Var}(\boldsymbol{\epsilon})=\boldsymbol{V}$, where $\boldsymbol{V}$ is a known $n \times n$ non-null, symmetric nonnegative-definite (n.n.d.) matrix. We denote the Gauss-Markov model defined above by $\{\boldsymbol{y}, \boldsymbol{X} \boldsymbol{\beta}, \boldsymbol{V}\}$, and we assume $\boldsymbol{y} \in \mathcal{C}(\boldsymbol{X}: \boldsymbol{V})$, where $C(\boldsymbol{X}: \boldsymbol{V})$ represents the column space of the partitioned matrix $(X: V)$.

Throughout the remainder of this paper, the notation $\mathbb{R}_{m \times n}$ represents the vector space of all $m \times n$ matrices over the real field $\mathbb{R}, \mathbb{R}_{n}^{S}$ denotes the set of $n \times n$ real symmetric matrices, $\mathbb{R}_{n}^{\geq}$represents the cone of all symmetric $n . n . d$. matrices in $\mathbb{R}_{n \times n}$, and $\mathbb{R}_{n}^{>}$denotes the interior of $\mathbb{R}_{n}^{\geq}$, which is the set of all symmetric positive-definite (p.d.) matrices. We use the notation $\boldsymbol{K}^{\prime}$ to denote the transpose of the real matrix $\boldsymbol{K} \in \mathbb{R}_{m \times n}$. Furthermore, we let $\boldsymbol{K}^{+} \in \mathbb{R}_{n \times m}$ and $\boldsymbol{K}^{-} \in \mathbb{R}_{n \times m}$ represent the Moore-Penrose pseudo-inverse and a generalized inverse of $\boldsymbol{K}$, respectively. Also, for $\boldsymbol{K} \in \mathbb{R}_{m \times n}$, we use the notation $\boldsymbol{P}_{\boldsymbol{K}}$ and $\boldsymbol{P}_{\boldsymbol{K}}^{\perp}$ to denote the orthogonal projection matrix onto $C(\boldsymbol{K})$ and $C(\boldsymbol{K})^{\perp}$, respectively.

Given $\boldsymbol{X}$, we define the ordinary least squares $(L S)$ estimator of $\boldsymbol{X} \boldsymbol{\beta}$ as

$$
\boldsymbol{X} \hat{\boldsymbol{\beta}}_{L S}=\boldsymbol{X}\left(\boldsymbol{X}^{\prime} \boldsymbol{X}\right)^{-} \boldsymbol{X}^{\prime} \boldsymbol{y} .
$$

Puntanen, Styan, and Isotalo (2011) have defined the best linear unbiased ( $B L U)$ estimator of $\boldsymbol{X} \boldsymbol{\beta}$ as

$$
\boldsymbol{X} \hat{\boldsymbol{\beta}}_{B L U}=\boldsymbol{X}\left(\boldsymbol{X}^{\prime} \boldsymbol{T}^{-} \boldsymbol{X}\right)^{-} \boldsymbol{X}^{\prime} \boldsymbol{T}^{-} \boldsymbol{y}
$$

where $\boldsymbol{T}=\boldsymbol{V}+\boldsymbol{X} \boldsymbol{U}^{\prime} \boldsymbol{X}$ and $\boldsymbol{U} \in \mathbb{R}_{n}^{S}$ is any $n \times n$ matrix such that $C(\boldsymbol{T})=C(\boldsymbol{X}: \boldsymbol{V})$. Puntanen et al. (2011) have defined the weighted least squares $(W L S)$ estimator as

$$
\boldsymbol{X} \hat{\boldsymbol{\beta}}_{W L S}=\boldsymbol{X}\left(\boldsymbol{X}^{\prime} \boldsymbol{V}^{+} \boldsymbol{X}\right)^{-} \boldsymbol{X}^{\prime} \boldsymbol{V}^{+} \boldsymbol{y} .
$$

In this paper we give two characterizations of the general n.n.d. error covariance structure $\boldsymbol{V}$ in the Gauss-Markov model $\{\boldsymbol{y}, \boldsymbol{X} \boldsymbol{\beta}, \boldsymbol{V}\}$ for which $\boldsymbol{X} \hat{\boldsymbol{\beta}}_{B L U}=\boldsymbol{X} \hat{\boldsymbol{\beta}}_{W L S}=\boldsymbol{X} \hat{\boldsymbol{\beta}}_{L S}$ where $\boldsymbol{y} \in C(\boldsymbol{X}: \boldsymbol{V})$. We define these covariance matrices to be $B L U$-WLS$L S$ estimator-equivalent (e.e.) covariance matrices. Specifically, in the first characterization we give a derivation of the 
explicit general n.n.d. $B L U$-WLS-LS e.e. covariance structure that is considerably more concise and straight-forward than the derivation given in Young, Odell and Hahn (2000). In the second characterization, we demonstrate that the MoorePenrose pseudo-inverse of the covariance matrices contained in the set of n.n.d. BLU-WLS-LS e.e. covariance structures are themselves elements of the set.

A large majority of previous work has focused on implicitly and explicitly characterizing the general covariance matrix $\boldsymbol{V}$ such that the $B L U$ and $L S$ estimators are equal. Puntanen and Styan (1989), Alalouf and Styan (1984), Tian and Wiens (2006), Proposition 10.1 in Puntanen et al. (2011), and numerous additional journal articles have presented many of these implicit characterizations.

However, we have found fewer results on explicit n.n.d. WLS-LS e.e. covariance structure characterizations. Plackett (1960), McElroy (1967), and Williams (1967) have derived sufficient (p.d.)WLS-LS e.e. covariance matrices. Additionally, for certain model matrices $\boldsymbol{X}$, Herzberg and Aleong (1985) have presented a sufficient $p . d$. WLS-LS e.e. covariance matrix, and Zyskind and Martin (1969), Searle (1994), and Tian and Wiens (2006) have presented several implicit WLS-LS e.e. covariance-structure characterizations. Results on both implicit and explicit characterizations of the general n.n.d. $B L U$ $W L S$ - $L S$ e.e. covariance structure for the Gauss-Markov model $\{\boldsymbol{y}, \boldsymbol{X} \boldsymbol{\beta}, \boldsymbol{V}\}$ appear to be more sparse. Herzberg and Aleong (1985) have presented two sufficient WLS-BLU-LS e.e. covariance matrices. Moreover, Baksalary and Kala (1983) have given an implicit characterization of the general n.n.d. e.e. covariance structure for $\boldsymbol{V}$, and Young et al. (2000) have explicitly characterized the general n.n.d. $B L U-W L S-L S$ e.e. covariance structure.

We have organized the remainder of the paper as follows. In Section 2 we state two lemmas that we use to derive the first of our two theorems. In Section 3 we present a new concise derivation our general n.n.d. BLU-WLS-LS e.e. dependency structure characterization for $\boldsymbol{V}$. We also demonstrate that the Moore-Penrose pseudo-inverse of elements contained in the set of n.n.d. $B L U$-WLS-LS e.e. covariance structures are themselves elements of this set. Last, in Section 4 we briefly summarize the two characterization results proven here.

\section{Preliminary Lemmas}

We next present two lemmas that we use in the proof of our first e.e.-covariance-structure characterization. The first lemma gives conditions for $\mathbf{V}$ such that $\boldsymbol{X} \hat{\boldsymbol{\beta}}_{B L U}=\boldsymbol{X} \hat{\boldsymbol{\beta}}_{W L S}=\boldsymbol{X} \hat{\boldsymbol{\beta}}_{L S}$. A proof of part a) is in the lemma in Zyskind (1967), a proof of b) is in Zyskind and Martin (1969), and a proof of part c) is in Theorem 2.2 of Baksalary and Kala (1983).

Lemma 1. For the Gauss-Markov model $\{\boldsymbol{y}, \boldsymbol{X} \boldsymbol{\beta}, \boldsymbol{V}\}$, we have

a) $\boldsymbol{X} \hat{\boldsymbol{\beta}}_{B L U}=\boldsymbol{X} \hat{\boldsymbol{\beta}}_{W L S}$ if and only if $C(\boldsymbol{X}) \subset C(\boldsymbol{V})$,

b) $\boldsymbol{X} \hat{\boldsymbol{\beta}}_{B L U}=\boldsymbol{X} \hat{\boldsymbol{\beta}}_{L S}$ if and only if $C(\boldsymbol{V} \boldsymbol{X}) \subset C(\boldsymbol{X})$, and

c) $\boldsymbol{X} \hat{\boldsymbol{\beta}}_{W L S}=\boldsymbol{X} \hat{\boldsymbol{\beta}}_{L S}$ if and only if $\boldsymbol{X} \hat{\boldsymbol{\beta}}_{B L U}=\boldsymbol{X} \hat{\boldsymbol{\beta}}_{W L S}=\boldsymbol{X} \hat{\boldsymbol{\beta}}_{L S}$.

In the second lemma, we state the general symmetric n.n.d. solution matrix to a particular homogeneous matrix that contains the column space of a specified matrix.

Lemma 2. Let $\boldsymbol{A} \in \mathbb{R}_{n \times q}$ such that $\operatorname{rank}(\boldsymbol{A})=k$, where $k \leq q<n$, and let $\mathscr{U}:=\left\{\boldsymbol{U} \in \mathbb{R}_{n}^{\geq}: C(\boldsymbol{A}) \subset \mathcal{C}(\boldsymbol{U})\right\}$. Then, a representation of the general n.n.d. solution to $\boldsymbol{P}_{\boldsymbol{A}} \boldsymbol{Z} \boldsymbol{P}_{\boldsymbol{A}}^{\perp}=\mathbf{0}$ such that $C(\boldsymbol{A}) \subset C(\boldsymbol{Z})$ is

$$
\boldsymbol{Z}=\boldsymbol{P}_{A} \boldsymbol{U}_{1} \boldsymbol{P}_{A}+\boldsymbol{P}_{A}^{\perp} \boldsymbol{U}_{2} \boldsymbol{P}_{A}^{\perp},
$$

where $\boldsymbol{U}_{1} \in \mathscr{U}$ and $\boldsymbol{U}_{2} \in \mathbb{R}_{n}^{\geq}$is arbitrary.

Proof. The proof is similar to the proof of Lemma 6 in Young et al. (2000).

\section{Main Results}

We now present a concise proof of the explicit characterization of the general n.n.d. BLU-WLS-LS e.e. covariance structure. The proof immediately below is considerably shorter and more direct than a previous proof given in Young et al. (2000).

Theorem 1. For the general Gauss-Markov model $\{\boldsymbol{y}, \boldsymbol{X} \boldsymbol{\beta}, \boldsymbol{V}\}$, we have $\boldsymbol{X} \hat{\boldsymbol{\beta}}_{B L U}=\boldsymbol{X} \hat{\boldsymbol{\beta}}_{W L S}=\boldsymbol{X} \hat{\boldsymbol{\beta}}_{L S}$ if and only if $\boldsymbol{V} \in \mathscr{V}$, where

$$
\mathscr{V}:=\left\{\boldsymbol{V} \in \mathbb{R}_{n}^{\geq}: \boldsymbol{V}=\boldsymbol{P}_{\boldsymbol{X}} \boldsymbol{W}_{1} \boldsymbol{P}_{\boldsymbol{X}}+\boldsymbol{P}_{\boldsymbol{X}}^{\perp} \boldsymbol{W}_{2} \boldsymbol{P}_{\boldsymbol{X}}^{\perp}\right\}
$$

with

$$
\boldsymbol{W}_{1} \in\left\{\boldsymbol{W} \in \mathbb{R}_{n}^{\geq}: C(\boldsymbol{X}) \subset C(\boldsymbol{W}\},\right.
$$

and $\boldsymbol{W}_{2} \in \mathbb{R}_{n}^{\geq}$is arbitrary. 
Proof. From Lemmas 1 and 2, we have that

$$
\begin{aligned}
\boldsymbol{X} \hat{\boldsymbol{\beta}}_{B L U}=\boldsymbol{X} \hat{\boldsymbol{\beta}}_{W L S}=\boldsymbol{X} \hat{\boldsymbol{\beta}}_{L S} & \Longleftrightarrow \boldsymbol{P}_{\boldsymbol{X}} \boldsymbol{V} \boldsymbol{X}=\boldsymbol{V} \boldsymbol{X} \text { and } \boldsymbol{P}_{\boldsymbol{V}} \boldsymbol{X}=\boldsymbol{X} \\
& \Longleftrightarrow \boldsymbol{P}_{\boldsymbol{X}} \boldsymbol{V} \boldsymbol{X}-\boldsymbol{V} \boldsymbol{X}=\mathbf{0} \text { and } \boldsymbol{P}_{\boldsymbol{V}} \boldsymbol{X}=\boldsymbol{X} \\
& \Longleftrightarrow\left(\boldsymbol{P}_{\boldsymbol{X}}-\boldsymbol{I}\right) \boldsymbol{V} \boldsymbol{P}_{\boldsymbol{X}}=\mathbf{0} \text { and } \boldsymbol{P}_{\boldsymbol{V}} \boldsymbol{X}=\boldsymbol{X} \\
& \Longleftrightarrow \boldsymbol{P}_{\boldsymbol{X}}^{\perp} \boldsymbol{V} \boldsymbol{P}_{\boldsymbol{X}}=\mathbf{0} \text { and } \boldsymbol{V} \boldsymbol{V}^{+} \boldsymbol{X}=\boldsymbol{X} \\
& \Longleftrightarrow \boldsymbol{V} \in \mathscr{V}, \text { where } \mathscr{V} \text { is given in (3). }
\end{aligned}
$$

Next, for the general Gauss-Markov model $\{\boldsymbol{y}, \boldsymbol{X} \boldsymbol{\beta}, \boldsymbol{V}\}$, we characterize the n.n.d. e.e. covariance matrices $\boldsymbol{V} \in \mathscr{V}$, defined in (3), by showing that for $\boldsymbol{V} \in \mathscr{V}$, the Moore-Penrose inverse $\boldsymbol{V}^{+}$has a particular form.

Theorem 2. For the general Gauss-Markov model $\{\boldsymbol{y}, \boldsymbol{X} \boldsymbol{\beta}, \boldsymbol{V}\}$, consider the covariance matrices $\boldsymbol{V} \in \mathscr{V}$ defined in (3). Then, $\boldsymbol{V} \in \mathscr{V}$ if and only if $\boldsymbol{V}^{+} \in \mathscr{V}$.

Proof. We first prove the necessity portion of Theorem 2. Let $\boldsymbol{V} \in \mathscr{V}$ be defined as in (3). In addition, let

$$
\boldsymbol{V}^{*}=\boldsymbol{P}_{\boldsymbol{X}} \boldsymbol{W}_{1}^{+} \boldsymbol{P}_{\boldsymbol{X}}+\boldsymbol{P}_{\boldsymbol{X}}^{\perp} \boldsymbol{W}_{2}^{+} \boldsymbol{P}_{\boldsymbol{X}}^{\perp}
$$

Then, using the definition of a Moore-Penrose pseudo-inverse and the facts that for $\boldsymbol{W}_{i} \in \mathbb{R}_{n}^{\geq}, \boldsymbol{P}_{\boldsymbol{X}} \boldsymbol{P}_{\boldsymbol{X}}^{\perp}=\boldsymbol{P}_{\boldsymbol{X}}^{\perp} \boldsymbol{P}_{\boldsymbol{X}}=\mathbf{0}$, and $\boldsymbol{P}_{\boldsymbol{X}} \boldsymbol{W}_{i}=\boldsymbol{W}_{i} \boldsymbol{P}_{\boldsymbol{X}}=\boldsymbol{W}_{i}, i=1,2$, we have

$$
\begin{aligned}
\boldsymbol{V} \boldsymbol{V}^{*} \boldsymbol{V} & =\left(\boldsymbol{P}_{\boldsymbol{X}} \boldsymbol{W}_{1} \boldsymbol{P}_{\boldsymbol{X}}+\boldsymbol{P}_{\boldsymbol{X}}^{\perp} \boldsymbol{W}_{2} \boldsymbol{P}_{\boldsymbol{X}}^{\perp}\right)\left(\boldsymbol{P}_{\boldsymbol{X}} \boldsymbol{W}_{1}^{+} \boldsymbol{P}_{\boldsymbol{X}}+\boldsymbol{P}_{\boldsymbol{X}}^{\perp} \boldsymbol{W}_{2}^{+} \boldsymbol{P}_{\boldsymbol{X}}^{\perp}\right)\left(\boldsymbol{P}_{\boldsymbol{X}} \boldsymbol{W}_{1} \boldsymbol{P}_{\boldsymbol{X}}+\boldsymbol{P}_{\boldsymbol{X}}^{\perp} \boldsymbol{W}_{2} \boldsymbol{P}_{\boldsymbol{X}}^{\perp}\right) \\
& \left.=\left(\boldsymbol{P}_{\boldsymbol{X}} \boldsymbol{W}_{1} \boldsymbol{P}_{\boldsymbol{X}}\right)\left(\boldsymbol{P}_{\boldsymbol{X}} \boldsymbol{W}_{1}^{+} \boldsymbol{P}_{\boldsymbol{X}}\right)\left(\boldsymbol{P}_{\boldsymbol{X}} \boldsymbol{W}_{1} \boldsymbol{P}_{\boldsymbol{X}}\right)+\left(\boldsymbol{P}_{\boldsymbol{X}}^{\perp} \boldsymbol{W}_{2} \boldsymbol{P}_{\boldsymbol{X}}^{\perp}\right)\left(\boldsymbol{P}_{\boldsymbol{X}}^{\perp} \boldsymbol{W}_{2}^{+} \boldsymbol{P}_{\boldsymbol{X}}^{\perp}\right) \boldsymbol{P}_{\boldsymbol{X}}^{\perp} \boldsymbol{W}_{2} \boldsymbol{P}_{\boldsymbol{X}}^{\perp}\right) \\
& =\left(\boldsymbol{P}_{\boldsymbol{X}} \boldsymbol{W}_{1} \boldsymbol{W}_{1}^{+} \boldsymbol{W}_{1} \boldsymbol{P}_{\boldsymbol{X}}\right)+\left(\boldsymbol{P}_{\boldsymbol{X}}^{\perp} \boldsymbol{W}_{2} \boldsymbol{W}_{2}^{+} \boldsymbol{W}_{2} \boldsymbol{P}_{\boldsymbol{X}}^{\perp}\right) \\
& =\left(\boldsymbol{P}_{\boldsymbol{X}} \boldsymbol{W}_{1} \boldsymbol{P}_{\boldsymbol{X}}+\boldsymbol{P}_{\boldsymbol{X}}^{\perp} \boldsymbol{W}_{2} \boldsymbol{P}_{\boldsymbol{X}}^{\perp}\right) \\
& =\boldsymbol{V} .
\end{aligned}
$$

Next, we have

$$
\begin{aligned}
\boldsymbol{V}^{*} \boldsymbol{V} \boldsymbol{V}^{*} & =\left(\boldsymbol{P}_{\boldsymbol{X}} \boldsymbol{W}_{1}^{+} \boldsymbol{P}_{\boldsymbol{X}}+\boldsymbol{P}_{\boldsymbol{X}}^{\perp} \boldsymbol{W}_{2}^{+} \boldsymbol{P}_{\boldsymbol{X}}^{\perp}\right)\left(\boldsymbol{P}_{\boldsymbol{X}} \boldsymbol{W}_{1} \boldsymbol{P}_{\boldsymbol{X}}+\boldsymbol{P}_{\boldsymbol{X}}^{\perp} \boldsymbol{W}_{2} \boldsymbol{P}_{\boldsymbol{X}}^{\perp}\right)\left(\boldsymbol{P}_{\boldsymbol{X}} \boldsymbol{W}_{1}^{+} \boldsymbol{P}_{\boldsymbol{X}}+\boldsymbol{P}_{\boldsymbol{X}}^{\perp} \boldsymbol{W}_{2}^{+} \boldsymbol{P}_{\boldsymbol{X}}^{\perp}\right) \\
& \left.=\left(\boldsymbol{P}_{\boldsymbol{X}} \boldsymbol{W}_{1}^{+} \boldsymbol{P}_{\boldsymbol{X}}\right)\left(\boldsymbol{P}_{\boldsymbol{X}} \boldsymbol{W}_{1} \boldsymbol{P}_{\boldsymbol{X}}\right)\left(\boldsymbol{P}_{\boldsymbol{X}} \boldsymbol{W}_{1}^{+} \boldsymbol{P}_{\boldsymbol{X}}\right)+\left(\boldsymbol{P}_{\boldsymbol{X}}^{\perp} \boldsymbol{W}_{2}^{+} \boldsymbol{P}_{\boldsymbol{X}}^{\perp}\right)\left(\boldsymbol{P}_{\boldsymbol{X}}^{\perp} \boldsymbol{W}_{2} \boldsymbol{P}_{\boldsymbol{X}}^{\perp}\right) \boldsymbol{P}_{\boldsymbol{X}}^{\perp} \boldsymbol{W}_{2}^{+} \boldsymbol{P}_{\boldsymbol{X}}^{\perp}\right) \\
& =\left(\boldsymbol{P}_{\boldsymbol{X}} \boldsymbol{W}_{1}^{+} \boldsymbol{W}_{1} \boldsymbol{W}_{1}^{+} \boldsymbol{P}_{\boldsymbol{X}}\right)+\left(\boldsymbol{P}_{\boldsymbol{X}}^{\perp} \boldsymbol{W}_{2}^{+} \boldsymbol{W}_{2} \boldsymbol{W}_{2}^{+} \boldsymbol{P}_{\boldsymbol{X}}^{\perp}\right) \\
& =\left(\boldsymbol{P}_{\boldsymbol{X}} \boldsymbol{W}_{1} \boldsymbol{P}_{\boldsymbol{X}}+\boldsymbol{P}_{\boldsymbol{X}}^{\perp} \boldsymbol{W}_{2} \boldsymbol{P}_{\boldsymbol{X}}^{\perp}\right) \\
& =\boldsymbol{V}^{*} .
\end{aligned}
$$

Third, let $\boldsymbol{W}_{1}$ be defined as in (4). Then, using the fact that $\boldsymbol{W}_{i} \boldsymbol{W}_{i}^{+}=\operatorname{big}\left(\boldsymbol{W}_{i} \boldsymbol{W}_{i}^{+}\right)^{\prime}=\boldsymbol{W}_{i}^{+} \boldsymbol{W}_{i}^{\prime}=\boldsymbol{W}_{i}^{+} \boldsymbol{W}_{i}, i=1,2$, we have

$$
\begin{aligned}
{\left[\boldsymbol{V} \boldsymbol{V}^{*}\right]^{\prime} } & =\left[\left(\boldsymbol{P}_{\boldsymbol{X}} \boldsymbol{W}_{1} \boldsymbol{P}_{\boldsymbol{X}}+\boldsymbol{P}_{\boldsymbol{X}}^{\perp} \boldsymbol{W}_{2} \boldsymbol{P}_{\boldsymbol{X}}^{\perp}\right)\left(\boldsymbol{P}_{\boldsymbol{X}} \boldsymbol{W}_{1}^{+} \boldsymbol{P}_{\boldsymbol{X}}+\boldsymbol{P}_{\boldsymbol{X}}^{\perp} \boldsymbol{W}_{2}^{+} \boldsymbol{P}_{\boldsymbol{X}}^{\perp}\right)\right]^{\prime} \\
& =\left(\boldsymbol{P}_{\boldsymbol{X}} \boldsymbol{W}_{1}^{+} \boldsymbol{P}_{\boldsymbol{X}}+\boldsymbol{P}_{\boldsymbol{X}}^{\perp} \boldsymbol{W}_{2}^{+} \boldsymbol{P}_{\boldsymbol{X}}^{\perp}\right)^{\prime}\left(\boldsymbol{P}_{\boldsymbol{X}} \boldsymbol{W}_{1} \boldsymbol{P}_{\boldsymbol{X}}+\boldsymbol{P}_{\boldsymbol{X}}^{\perp} \boldsymbol{W}_{2} \boldsymbol{P}_{\boldsymbol{X}}^{\perp}\right)^{\prime} \\
& =\left(\boldsymbol{P}_{\boldsymbol{X}} \boldsymbol{W}_{1}^{+} \boldsymbol{P}_{\boldsymbol{X}}\right)\left(\boldsymbol{P}_{\boldsymbol{X}} \boldsymbol{W}_{1} \boldsymbol{P}_{\boldsymbol{X}}\right)+\left(\boldsymbol{P}_{\boldsymbol{X}}^{\perp} \boldsymbol{W}_{2} \boldsymbol{P}_{\boldsymbol{X}}^{\perp}\right)\left(\boldsymbol{P}_{\boldsymbol{X}}^{\perp} \boldsymbol{W}_{2}^{+} \boldsymbol{P}_{\boldsymbol{X}}^{\perp}\right) . \\
& =\boldsymbol{P}_{\boldsymbol{X}} \boldsymbol{W}_{1}^{+} \boldsymbol{W}_{1} \boldsymbol{P}_{\boldsymbol{X}}+\boldsymbol{P}_{\boldsymbol{X}}^{\perp} \boldsymbol{W}_{2}^{+} \boldsymbol{W}_{2} \boldsymbol{P}_{\boldsymbol{X}}^{\perp} \\
& =\boldsymbol{P}_{\boldsymbol{X}} \boldsymbol{W}_{1} \boldsymbol{W}_{1}^{+} \boldsymbol{P}_{\boldsymbol{X}}+\boldsymbol{P}_{\boldsymbol{X}}^{\perp} \boldsymbol{W}_{2} \boldsymbol{W}_{2}^{+} \boldsymbol{P}_{\boldsymbol{X}}^{\perp} \\
& =\left(\boldsymbol{P}_{\boldsymbol{X}} \boldsymbol{W}_{1} \boldsymbol{P}_{\boldsymbol{X}}\right)\left(\boldsymbol{P}_{\boldsymbol{X}} \boldsymbol{W}_{1}^{+} \boldsymbol{P}_{\boldsymbol{X}}\right)+\left(\boldsymbol{P}_{\boldsymbol{X}}^{\perp} \boldsymbol{W}_{2} \boldsymbol{P}_{\boldsymbol{X}}^{\perp}\right)\left(\boldsymbol{P}_{\boldsymbol{X}}^{\perp} \boldsymbol{W}_{2}^{+} \boldsymbol{P}_{\boldsymbol{X}}^{\perp}\right) . \\
& =\left(\boldsymbol{P}_{\boldsymbol{X}} \boldsymbol{W}_{1} \boldsymbol{P}_{\boldsymbol{X}}+\boldsymbol{P}_{\boldsymbol{X}}^{\perp} \boldsymbol{W}_{2} \boldsymbol{P}_{\boldsymbol{X}}^{\perp}\right)\left(\boldsymbol{P}_{\boldsymbol{X}} \boldsymbol{W}_{1} \boldsymbol{P}_{\boldsymbol{X}}+\boldsymbol{P}_{\boldsymbol{X}}^{\perp} \boldsymbol{W}_{2} \boldsymbol{P}_{\boldsymbol{X}}^{\perp}\right) . \\
& =\boldsymbol{V} \boldsymbol{V}^{*} .
\end{aligned}
$$

Last, again using the fact that $\boldsymbol{W}_{i} \boldsymbol{W}_{i}^{+}=\boldsymbol{W}_{i}^{+} \boldsymbol{W}_{i}, i=1,2$, we have that

$$
\begin{aligned}
{\left[\boldsymbol{V}^{*} \boldsymbol{V}\right]^{\prime} } & =\left[\left(\boldsymbol{P}_{\boldsymbol{X}} \boldsymbol{W}_{1}^{+} \boldsymbol{P}_{\boldsymbol{X}}+\boldsymbol{P}_{\boldsymbol{X}}^{\perp} \boldsymbol{W}_{2}^{+} \boldsymbol{P}_{\boldsymbol{X}}^{\perp}\right)\left(\boldsymbol{P}_{\boldsymbol{X}} \boldsymbol{W}_{1} \boldsymbol{P}_{\boldsymbol{X}}+\boldsymbol{P}_{\boldsymbol{X}}^{\perp} \boldsymbol{W}_{2} \boldsymbol{P}_{\boldsymbol{X}}^{\perp}\right)\right]^{\prime} \\
& =\left(\boldsymbol{P}_{\boldsymbol{X}} \boldsymbol{W}_{1} \boldsymbol{P}_{\boldsymbol{X}}+\boldsymbol{P}_{\boldsymbol{X}}^{\perp} \boldsymbol{W}_{2} \boldsymbol{P}_{\boldsymbol{X}}^{\perp}\right)^{\prime}\left(\boldsymbol{P}_{\boldsymbol{X}} \boldsymbol{W}_{1}^{+} \boldsymbol{P}_{\boldsymbol{X}}+\boldsymbol{P}_{\boldsymbol{X}}^{\perp} \boldsymbol{W}_{2}^{+} \boldsymbol{P}_{\boldsymbol{X}}^{\perp}\right)^{\prime} \\
& =\left(\boldsymbol{P}_{\boldsymbol{X}} \boldsymbol{W}_{1} \boldsymbol{P}_{\boldsymbol{X}}\right)\left(\boldsymbol{P}_{\boldsymbol{X}} \boldsymbol{W}_{1}^{+} \boldsymbol{P}_{\boldsymbol{X}}\right)+\left(\boldsymbol{P}_{\boldsymbol{X}}^{\perp} \boldsymbol{W}_{2} \boldsymbol{P}_{\boldsymbol{X}}^{\perp}\right)\left(\boldsymbol{P}_{\boldsymbol{X}}^{\perp} \boldsymbol{W}_{2}^{+} \boldsymbol{P}_{\boldsymbol{X}}^{\perp}\right) . \\
& =\boldsymbol{P}_{\boldsymbol{X}} \boldsymbol{W}_{1} \boldsymbol{W}_{1}^{+} \boldsymbol{P}_{\boldsymbol{X}}+\boldsymbol{P}_{\boldsymbol{X}}^{\perp} \boldsymbol{W}_{2} \boldsymbol{W}_{2}^{+} \boldsymbol{P}_{\boldsymbol{X}}^{\perp} \\
& =\boldsymbol{P}_{\boldsymbol{X}} \boldsymbol{W}_{1}^{+} \boldsymbol{W}_{1} \boldsymbol{P}_{\boldsymbol{X}}+\boldsymbol{P}_{\boldsymbol{X}}^{\perp} \boldsymbol{W}_{2}^{+} \boldsymbol{W}_{2} \boldsymbol{P}_{\boldsymbol{X}}^{\perp} \\
& =\left(\boldsymbol{P}_{\boldsymbol{X}} \boldsymbol{W}_{1}^{+} \boldsymbol{P}_{\boldsymbol{X}}\right)\left(\boldsymbol{P}_{\boldsymbol{X}} \boldsymbol{W}_{1} \boldsymbol{P}_{\boldsymbol{X}}\right)+\left(\boldsymbol{P}_{\boldsymbol{X}}^{\perp} \boldsymbol{W}_{2}^{+} \boldsymbol{P}_{\boldsymbol{X}}^{\perp}\right)\left(\boldsymbol{P}_{\boldsymbol{X}}^{\perp} \boldsymbol{W}_{2} \boldsymbol{P}_{\boldsymbol{X}}^{\perp}\right) . \\
& =\left(\boldsymbol{P}_{\boldsymbol{X}} \boldsymbol{W}_{1}^{+} \boldsymbol{P}_{\boldsymbol{X}}+\boldsymbol{P}_{\boldsymbol{X}}^{\perp} \boldsymbol{W}_{2} \boldsymbol{P}_{\boldsymbol{X}}^{\perp}\right)\left(\boldsymbol{P}_{\boldsymbol{X}} \boldsymbol{W}_{1} \boldsymbol{P}_{\boldsymbol{X}}+\boldsymbol{P}_{\boldsymbol{X}}^{\perp} \boldsymbol{W}_{2} \boldsymbol{P}_{\boldsymbol{X}}^{\perp}\right) . \\
& =\boldsymbol{V}^{*} \boldsymbol{V} .
\end{aligned}
$$


Hence, $\boldsymbol{V}^{*}=\boldsymbol{V}^{+}$. The sufficiency portion of the proof is similar to the necessity portion because of the facts that $\left[\boldsymbol{V}^{+}\right]^{+}=\boldsymbol{V}$ and $\left[\boldsymbol{W}_{i}^{+}\right]^{+}=\boldsymbol{W}_{i}, i=1,2$.

The following corollary, which follows directly from Theorems 1 and 2, gives several implicit characterizations of the general n.n.d. BLU-WLS-LS e.e. dependency matrix.

Corollary. Let $\mathscr{V}$ be defined as in (3). Then, $\boldsymbol{V} \in \mathscr{V}$ if and only if $C(\boldsymbol{X}) \subset \mathcal{C}(\boldsymbol{V})$, and

a) $\boldsymbol{P}_{X}^{\perp} \boldsymbol{V} \boldsymbol{P}_{X}^{\perp}=\boldsymbol{P}_{X}^{\perp} \boldsymbol{V}$

b) $\boldsymbol{P}_{X}^{\perp} \boldsymbol{V}^{+} \boldsymbol{P}_{X}^{\perp}=\boldsymbol{P}_{X}^{\perp} \boldsymbol{V}^{+}$

c) $\boldsymbol{P}_{X} V \boldsymbol{P}_{X}=\boldsymbol{P}_{X} \boldsymbol{V}$

d) $\boldsymbol{P}_{\boldsymbol{X}} \boldsymbol{V}^{+} \boldsymbol{P}_{\boldsymbol{X}}=\boldsymbol{P}_{\boldsymbol{X}} \boldsymbol{V}^{+}$

e) $\boldsymbol{P}_{\boldsymbol{X}} \boldsymbol{V}=\boldsymbol{V} \boldsymbol{P}_{\boldsymbol{X}}$

f) $\boldsymbol{P}_{\boldsymbol{X}} \boldsymbol{V}^{+}=\boldsymbol{V}^{+} \boldsymbol{P}_{\boldsymbol{X}}$

g) $\boldsymbol{P}_{X}^{\perp} \boldsymbol{V}=\boldsymbol{V} \boldsymbol{P}_{X}^{\perp}$

h) $\boldsymbol{P}_{\boldsymbol{X}}^{\perp} \boldsymbol{V}^{+}=\boldsymbol{V}^{+} \boldsymbol{P}_{\boldsymbol{X}}^{\perp}$.

\section{Summary}

We have derived two explicit characterizations of the general n.n.d. e.e. covariance structure such that $\boldsymbol{X} \hat{\boldsymbol{\beta}}_{B L U}=\boldsymbol{X} \hat{\boldsymbol{\beta}}_{W L S}=$ $\boldsymbol{X} \hat{\boldsymbol{\beta}}_{L S}$. Theorem 1 provides a brief derivation of the explicit general n.n.d. BLU-WLS-LS e.e. dependency structure that considerably shortens a proof given in Young et al. (2000). Theorem 2 presents a second characterization of the general n.n.d. BLU-WLS-LS e.e. covariance matrix $\mathbf{V}$ in which we prove that $\mathbf{V}$ and $\mathbf{V}^{+}$have the same general structure. Last, we give some implicit characterizations of the general n.n.d. e.e. covariance matrices such that $\boldsymbol{X} \hat{\boldsymbol{\beta}}_{B L U}=\boldsymbol{X} \hat{\boldsymbol{\beta}}_{W L S}=\boldsymbol{X} \hat{\boldsymbol{\beta}}_{L S}$.

\section{Acknowledgements}

We wish to thank Joy L. Young for her help in the writing of this paper.

\section{References}

Alalouf, I. S., \& Styan, G. P. (1984). Characterizations of the conditions for the ordinary least squares estimator to be best linear unbiased. In Y. P. Chaubey, \& T. D. Dwivedi (Eds.), Topics in Applied Statistics, Dept. of Mathematics, Concordia Univ., Montreal, (pp. 331-344).

Baksalary, J., \& Kala, R. (1983). On equalities between BLUEs, WLSEs, and SLSEs. Canadian Journal of Statistics, 11, 119-123. https://doi.org/10.2307/3314978

Herzberg, A. M., \& Aleong, J. (1985). Further conditions on the equivalence of ordinary least squares and weighted least squares estimators with examples. In J. Lanke \& G. Lindgren (Eds.), In Contributions to Probability and Statistics in Honour of Gunnar Blom, University of Lund, (pp. 127-142).

McElroy, F. W. (1967). A necessary and sufficient condition that ordinary least-squares estimators be best linear unbiased. Journal of the American Statistical Association, 62, 1302-1304. https://doi.org/10.1080/01621459.1967.10500935

Plackett, R. L. (1960). Principles of Regression Analysis. Clarendon Press, Oxford.

Puntanen, S., \& Styan, G. P. (1989). The equality of the ordinary least squares estimator and the best linear unbiased estimator. American Statistician, 43, 153-161. https://doi.org/10.1080/00031305.1989.10475644

Puntanen, S., Styan, G. P., \& Isotalo, J. (2011). Matrix Tricks for Linear Models. Springer, New York.

Searle, S. R. (1994). Extending some results and proofs for the singular linear model. Linear Algebra Appl., 210, $139-151$. https://doi.org/10.1016/0024-3795(94)90469-3

Tian, Y., \& Wiens, D. P. (2006). On equality and proportionality of ordinary least squares, weighted least squares, and best linear unbiased estimators in the general linear model. Statistics and Probability Letters, 76, $1265-1272$. https://doi.org/10.1016/j.spl.2006.01.005

Williams, J. S. (1967). The variance of weighted estimators. Journal of the American Statistical Association, 62, 12901301. https://doi.org/10.1080/01621459.1967.10500934 
Young, D. M., Odell, P. L., \& Hahn, W. (2000). Nonnegative-definite covariance structures for which the BLU, WLS, and LS estimators are equal. Statistics and Probability Letters, 49, 271-276. https://doi.org/10.1016/S0167-7152(00)000572

Zyskind, G. (1967). On canonical forms, nonnegative covariance matrices and best and simple least squares linear estimators in the linear model. Annals of Mathematical Statistics, 38, 1092-1109. https://doi.org/10.1214/aoms/1177698779

Zyskind, G., \& Martin, F. B. (1969). On best linear estimation and a general Gauss-Markov theorem in linear model with arbitrary nonnegative covariance structure. SIAM Journal on Applied Mathematics, 17, 1190-1202. https://doi.org/10$.1137 / 0117110$

\section{Copyrights}

Copyright for this article is retained by the author(s), with first publication rights granted to the journal.

This is an open-access article distributed under the terms and conditions of the Creative Commons Attribution license (http://creativecommons.org/licenses/by/4.0/). 